\title{
Genotypic characterization of Malaysian human isolates of Streptococcus pneumoniae from carriage and clinical sources
}

\begin{abstract}
This study characterized carriage and clinical pneumococcal isolates for serotypes, penicillin susceptibility, virulence genes and restriction fragment length polymorphism (RFLP) pattern of penicillin binding protein (PBP) genes. DNA fingerprint of isolates was generated by BOX-PCR. Majority of serotypes were $23 \mathrm{~F}$ followed by 19F, 19A and 6A. Twenty-four percent of isolates were penicillin non-susceptible (PNSP). All of the targeted virulence genes were detected in all isolates with the exception of pili; $20.6 \%(\mathrm{n}=22)$ for PI-1 and $14.0 \%(\mathrm{n}=15)$ for PI-2. Of the 13 isolates which carried both PI-1 and PI-2, 10 were of clinical origin. Digested pbp-DNA produced three PBP-RFLP profiles for pbp1a (A1 to A3), six profiles for pbp2b (B1 to B6) and seven for pbp2x (X1 to X7) mostly in PNSPs. Based on BOX-PCR analysis, the majority of isolates were genetically diverse with a small number of potentially related isolates carrying pili genes. No obvious genotypic association was observed pertaining to carriage and clinical origin of isolates.
\end{abstract}

Keyword: Streptococcus pneumoniae; Serotype; Virulence; Pili; Vaccine; PBP-RFLP; BOXPCR 